\title{
Review Article \\ Subclinical Detection of Diabetic Cardiomyopathy with MicroRNAs: Challenges and Perspectives
}

\author{
Luis E. León, ${ }^{1}$ Sweta Rani, ${ }^{2}$ Mauricio Fernandez, ${ }^{3}$ \\ Martín Larico, ${ }^{3}$ and Sebastián D. Calligaris ${ }^{4}$ \\ ${ }^{1}$ Centro de Genética y Genómica, Facultad de Medicina, Clínica Alemana Universidad del Desarrollo, 7710162 Santiago, Chile \\ ${ }^{2}$ Regenerative Medicine Institute (REMEDI), National University of Ireland, Galway, Ireland \\ ${ }^{3}$ Clínica Alemana, 7650568 Santiago, Chile \\ ${ }^{4}$ Centro de Medicina Regenerativa, Facultad de Medicina, Clínica Alemana-Universidad del Desarrollo, 7710162 Santiago, Chile
}

Correspondence should be addressed to Sebastián D. Calligaris; scalligaris@udd.cl

Received 1 June 2015; Revised 17 July 2015; Accepted 26 July 2015

Academic Editor: Dirk Westermann

Copyright (C) 2016 Luis E. León et al. This is an open access article distributed under the Creative Commons Attribution License, which permits unrestricted use, distribution, and reproduction in any medium, provided the original work is properly cited.

\begin{abstract}
The prevalence of cardiac diabetic diseases has been increased around the world, being the most common cause of death and disability among diabetic patients. In particular, diabetic cardiomyopathy is characterized with a diastolic dysfunction and cardiac remodelling without signs of hypertension and coronary artery diseases. In an early stage, it is an asymptomatic disease; however, clinical studies demonstrate that diabetic myocardia are more vulnerable to injury derived by acute myocardial infarct and are the worst prognosis for rehabilitation. Currently, biochemical and imaging diagnostic methods are unable to detect subclinical manifestation of the disease (prior to diastolic dysfunction). In this review, we elaborately discuss the current scientific evidences to propose circulating microRNAs as promising biomarkers for early detection of diabetic cardiomyopathy and, then, to identify patients at high risk of diabetic cardiomyopathy development. Moreover, here we summarise the research strategies to identify miRNAs as potential biomarkers, present limitations, challenges, and future perspectives.
\end{abstract}

\section{Introduction}

The global prevalence of diabetes was estimated as 387 million people in 2014 and expected to increase to 592 million by 2035. Diabetes is a chronic disease that leads to multisystem complications such as nephropathy, retinopathy, neuropathy, and cardiovascular diseases. In 2014 alone about 4.9 million people died due to diabetes-related diseases, cardiovascular disease being the most common cause of death and disability among people with diabetes [1].

Diabetes slowly reduces heart function, (1) promoting the generation of atheroma in coronary arteries (atherosclerosis), reducing oxygen and nutrient supply to cardiac cells [2], (2) impairing autonomic nerve fibres that innervate the blood vessels and heart that produce abnormalities in control heart rate (arrhythmia) and vessels dynamics (cardiac autonomic neuropathy) [3], and (3) reducing contractile capacity of muscle cells and decreasing capillarity irrigation of myocardium (diabetic cardiomyopathy) [4]. While atherosclerosis is well known to increase the risk of heart failure through an episode of ischemia, the weakening of the cardiac fibres by the silent progression of diabetic cardiomyopathy is not accounted due to technical limitations in its subclinical detection. Population-based cohort studies reported by From et al. suggested that patients with heart failure and diabetes but no atherosclerosis had a higher risk of death [5]. In fact, Shah et al. reported that diabetes is related to a higher risk of heart failure hospitalization or death, independently of the left ventricular efficiency fraction levels in patients after a myocardial infarct event; that is, diabetic patients have less capacity to recuperate cardiac functions to normal levels with respect to nondiabetic patients [6].

Diabetic cardiomyopathy was defined first in 1972 as a heart failure without signs of coronary artery disease, 
hypertension, or valvular or congenital heart disease by Rubler et al. [7]. In spite of the differences in etiology and metabolic profile, many pathophysiologic features of this cardiomyopathy are shared by diabetes mellitus type 1 (DMT1) and diabetes mellitus type 2 (DMT2) [8].

Considering these common pathophysiological mechanisms, in a prediabetic and diabetic state, plasma level of free fatty acid (FFA) is increased, producing an augmented uptake, accumulation, and oxidation of FFA in the cardiomyocytes. The excess of FFA utilization generates a concomitant suppression of glucose oxidation by the indirect inhibition of the pyruvate dehydrogenase and a downregulation of glucose transporters 1 and 4 (GLUT1 and GLUT4), establishing a metabolic derangement. The deficiency of glycolysis intermediates decreases the mitochondrial ATP synthesis by oxidative phosphorylation. To compensate this limitation and to attend the high ATP requirement of the heart, $\beta$-oxidation of FFA has a prominent role in increasing oxygen consumption and reactive oxygen species (ROS), which upregulates the uncoupling proteins expression to balance the proton transmembrane gradient needed for ATP synthesis [9]. The excessive ROS production stimulates apoptotic signals by ceramide generation and mitochondrial cytochrome $c$ release [10]. Besides the deficiency of energy production, the excitation contraction coupling, essential for cardiac contraction, is altered by impaired intracellular $\mathrm{Ca}^{+2}$ handling [9].

The mitochondrial oxidative stress and "glycolated" tissue state produces an endothelial dysfunction and a proinflammatory microenvironment that stimulated the infiltration of macrophages and leukocytes that aggravates heart inflammation and tissue damage [9]. The remodelling of extracellular matrix is characterized by an interstitial and perivascular fibrosis and abnormalities in microvasculature [11]. In a latent subclinical period, metabolic disturbances and structural abnormalities lead to a diastolic dysfunction, which subsequently progresses to left ventricular hypertrophy, contractility reserve impairment, and, eventually, a systolic dysfunction [12].

Currently, therapy for diabetic cardiomyopathy is based on glycaemia control and hypoglycaemic drugs administration and changes in lifestyle (for DMT2), which delay the progression to heart failure but not revert it; however, therapy efficacy can be improved with earliest detection [13]. In this review we discuss the usefulness and limitations of the current methods used in the clinic to diagnose diabetic cardiomyopathy. We summarize the scientific evidences to propose miRNAs as new generation of biomarkers at subclinical stages of this disease by reflexing in biofluids the myocardial metabolic derangement before cardiac dysfunction.

\section{Diagnosis of Diabetic Cardiomyopathy}

Diabetic cardiomyopathy detection is a challenge in the clinical practice due to lack of any specific pathognomonic histologic changes or imaging characteristics. However, diastolic dysfunction and cardiac hypertrophy (measured by tissue Doppler echocardiography) in the absence of coronary artery disease and hypertension have been considered the two principal hallmarks to propose a diagnosis of diabetic cardiomyopathy in asymptomatic diabetic patients $[14,15]$.

Imaging diagnosis techniques were also used to detect myocardial metabolic changes in diabetic patients. McGavock et al. were able to detect the excessive storage of lipid in myocardium of patients with prediabetic stage using proton magnetic resonance spectroscopy, proposing this finding as an indicator of heart failure risk. However, they did not demonstrate a correlation between myocardial lipid content and cardiac function [16]. In addition, positron emission tomography was used to establish an association between myocardial metabolic derangement and early manifestation of diastolic function impairment with negative results [17].

Regarding serological biomarkers, natriuretic peptides and brain natriuretic peptide (BNP) in particular were proposed as suitable biomarkers for diastolic dysfunction in diabetic patients [18]. However, the utility of this molecule is controversial because plasma BNP rise is associated with excessive stretching of heart muscle cells, a condition associated with several cardiac diseases [19]. Troponins plasma concentration is associated with the magnitude of cardiomyocyte death, resulting in a biomarker of heart damage without any specificity of the cardiac disease etiology. In addition, troponins in plasma have a short half-life and are usually used to predict and establish heart failure [20].

At present, neither a laboratory test nor imaging techniques appear to be useful in diagnosing diabetic cardiomyopathy apart from diastolic dysfunction and to predict the risk of heart failure, excluding coronary artery disease, hypertension, or congenital heart failure [13]. Therefore, regarding the subclinical detection of diabetic cardiomyopathy, regulators of metabolic changes in the heart, as mentioned above, also present in biofluids, could be appropriate candidates as biomarkers. In the last 6 years, a large number of publications have been reported with promising results about the correlation of diseases manifestation and miRNAs (showing potential as a new class of biomarker) [21].

\section{3. miRNAs Role in Diabetic Cardiomyopathy}

As we previously described, changes in gene expression of key molecules involved in the pathogenesis of diabetic cardiomyopathy can be influenced by environmental factors, for instance, high fat diets, tobacco smoke, or epigenetic factors [22]. At present, epigenetic studies describe three mechanisms to link the type of exposure with cellular gene expression response: DNA methylation, histone modification, and miRNA expression [23].

MicroRNAs or miRNAs are small noncoding RNA molecules ( $\approx 22$ nucleotides) which downregulate gene expression by a posttranscriptional mechanism controlling approximately $30 \%$ of all protein-coding genes of mammalian genome $[24,25]$. During the past 7 years, researchers have identified several miRNAs and their specific mRNA targets altered in diabetic cardiomyopathy using experimental models at preclinic level, demonstrating the significant role of miRNAs in the progression of diabetic heart complication (Table 1). Human biopsies of diabetic heart showed an upregulation of miR-223 with an inhibition of GLUT4 gene expression, reducing glucose uptake. This miRNA-mRNA 


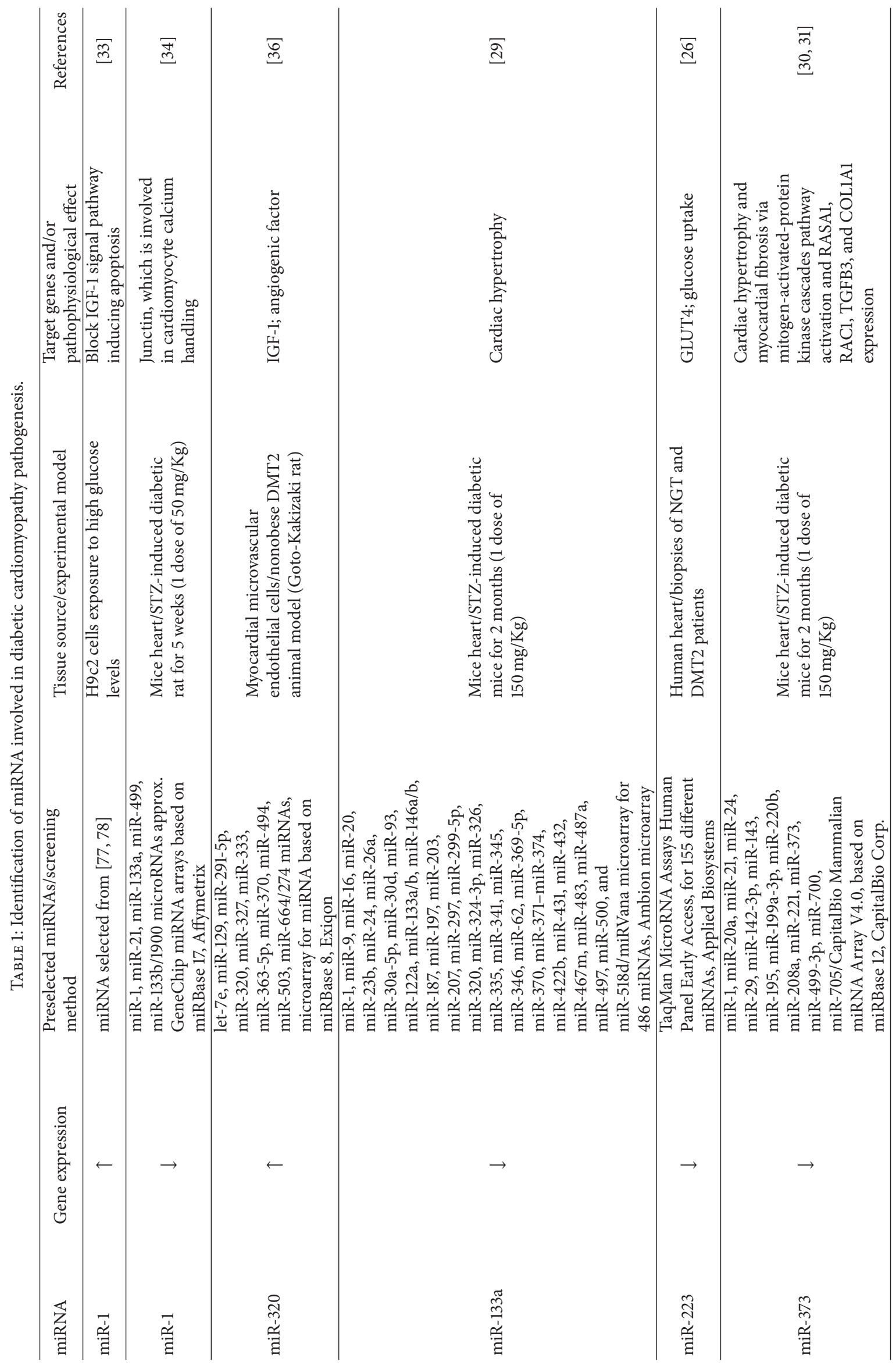




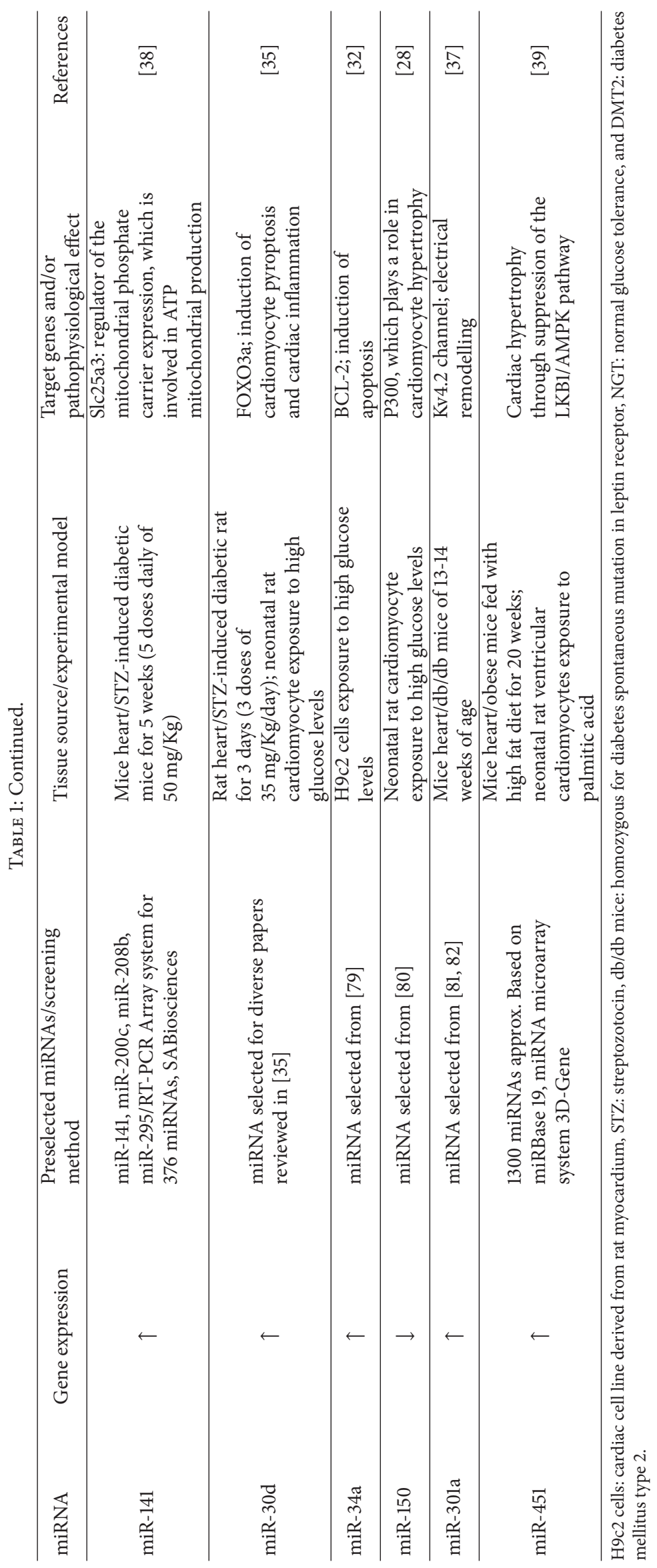


interaction was confirmed using neonatal rat cardiomyocyte [26]. El Azzouzi et al. reported that miR-199a/miR-214 cluster downregulated the peroxisome proliferator-activated receptor $\delta$ gene expression, which is a critical regulator of energy metabolism switch between fatty acid oxidation and glycolysis, impairing mitochondrial fatty acid oxidation [27]. Cardiomyocyte hypertrophy induced by exposing neonatal rat cardiomyocytes to high levels of glucose identified significantly reduced expression of three miRNAs (miR-150, miR133a, and miR-373) involved in cardiac hypertrophy process [28-30]. Diao et al. identified sixteen microRNAs differentially expressed in hearts of DMT1 animal model induced by streptozotocin and proposed 4 gene targets (Rasal, Racl, $T g f b 3$, and CollA1) associated with cardiac hypertrophy and myocardial fibrosis [31]. Upregulation of miR-34a and miR-1, induced by high glucose exposure, decreased $\mathrm{Bcl}-2$ and Igf1 gene expression, respectively, promoting apoptosis in $\mathrm{H} 9 \mathrm{c} 2$ cells $[32,33]$. Regarding cardiomyocyte $\mathrm{Ca}^{+2}$ handling during contractility-relaxation cycle, Yildirim et al. demonstrated that myocardial miR-1 downregulation produces an increase of junctin levels in streptozotocin-induced diabetic mice. Junctin is a component of the ryanodine receptor $\mathrm{Ca}^{+2}$ release channel complex in sarcoplasmic reticulum [34]. These data suggest that miR-1 has many target genes (e.g., Igf-1 and Junctin) and its regulation could depend on the experimental model, indicating the nonspecificity of this miRNA in diabetic cardiomyopathy. Upregulation of miR30d promoted cardiomyocyte pyroptosis (the proinflammatory programmed cell death) in a DMT1 animal model, via the repression of foxo3a and apoptosis repressor with caspase recruitment domain expression and, consequently, the activation of caspase- 1 and secretion of proinflammatory cytokines (IL-1 $\beta$ and IL-18) [35]. Using myocardial microvascular endothelial cells from a nonobese DMT2 animal model (Goto-Kakizaki rat), upregulation of miR-320 was reported that reduced Igf-1 gene expression decreasing angiogenic response to diabetes-derived microvascular injury [36]. miR301 upregulation alters the voltage-gated potassium channel in diabetic heart of $d b / d b$ mice (animal model for DMT2), generating an electrical remodelling [37]. miR-141 upregulation in heart of diabetic mice (induced with streptozotocin) reduced the gene expression of Slc25a3 (inner mitochondrial phosphate transporter), resulting in a decreased ATP production [38]. Recently, Kuwabara et al. showed that miR451 exacerbates lipotoxicity in cardiac myocytes and cardiac hypertrophy in obese mice fed with high fat diet for 20 weeks (a physiological obese animal model for a prediabetic state of DMT2) through the direct interaction with Cab39, scaffold protein of liver kinase B1 (LKB1), which suppress the LKB1/AMPK pathway. As AMP-activated protein kinase (AMPK) is a major cellular response of energy availability, its suppression reduced the cardiac functional reserve [39]. We summarised the miRNAs in pathological mechanism of diabetes cardiomyopathy in Table 1.

\section{4. miRNAs as Biomarkers}

Besides the intracellular functions of miRNAs, recent studies demonstrated that miRNAs are also paracrine mediators of cell-to-cell communication transported via microvesicles called exosomes. Cardiac cell communication via exosomes in healthy and pathological conditions is an emerging research field for understanding the development of cardiac diseases [40]. Malik et al. published that oxidative stress or hypoxia/reoxygenation transition stimulated cardiomyocyte to secrete exosomes containing mRNAs and miRNAs [41]. In addition, external cell signalling, as growth factor stimulation, can regulate the transcriptional contents of secreted exosomes in cardiomyocytes [42]. Waldenström et al. reported that exosomes released from cardiomyocytes affect gene expression in fibroblast [43]. Reciprocally, cardiac fibroblast was found to release miR-21 via exosomes and was associated with cardiomyocyte hypertrophy [44]. According to the current scientific evidences, both miRNA composition and quantity could be considered a reflex of metabolic or differentiated state of exosome-producing cells. Circulating exosomes can be identified in all biofluids, particularly in plasma or serum [45], and, therefore, becoming an attractive tool for analytical studies and subsequent diagnosis of the diseases.

Moreover, circulating miRNAs in plasma are also transported and delivered to recipient cells on circulating highdensity lipoprotein (HDL) [46]. HDL has a crucial role in the progression of cardiolipid-metabolism modifications occurring in diabetic cardiomyopathy development; for instance, in cardiomyocyte FFA oxidation via AMP-kinase activation and its accumulation prevents lipotoxicity in diabetic heart [47]. The discovery of miRNA participation in the regulation of lipoprotein synthesis, composition, transport, and degradation has provided new targets for therapy to improve the cardioprotective properties of HDL, particularly in coronary artery disease due to HDL regulation of cholesterol homeostasis [48]. Taking that into account, circulating miRNA in HDL complex could also be an indicator of metabolic changes in lipid-tissue accumulation diseases such as diabetic cardiomyopathy.

A third class of circulating miRNAs bind to soluble proteins called Argonautes, which are key players in all smallRNA-guided gene silencing processes [49]. Biophysics studies demonstrated that extracellular miRNA circulating in the bloodstream is remarkably stable, in spite of being presented in an RNAse-rich environment, due to its encapsulation in microvesicles/exosomes or its binding to the proteins [50]. This known stability of miRNA-exosomes and miRNAprotein complex is another relevant characteristic suitable for biomarkers.

\section{Evidences of miRNA Profiling for Diabetic Cardiomyopathy}

Identification of miRNA associated with diabetes and its complications in humans escalated with the possibility of screening multiple miRNAs simultaneously using profiling techniques including microarray and Next Generation Sequencing (NGS), in damaged or injury tissues of diabetes animal models due to miRNA-mRNA interaction found to be conserved between most mammalians [51]. The gene target identification of the selected miRNAs has been 
studied in vitro using neonatal rat cardiomyocytes where the miRNA-mRNA interaction is correlated with changes in cell phenotype. The process of gene targets identification is accelerated by bioinformatics techniques such as TargetScan, DIANA-mirExTra, PITA, miRNADA, miRDB, and PICTAR that reduce the number of possibilities to test experimentally [52-54]. In this context a good resource that integrates all these tools is miRWalk database that allows choosing between predicted and validated experimental miRNA-mRNA interactions [55].

The discovery of placental miRNA in maternal plasma in 2008 [56], along with the nascent hypothesis of its role in the cell-to-cell communication, has promoted the study of miRNAs as biomarkers in cancer through the specific secretion via exosomes by tumour cells [21]. In the same year, Lawrie et al. reported elevated levels of tumour-associated miRNAs in serum of patients suffering from diffuse large B-cell lymphoma [57]. Several clinical studies are also performed proposing use of miRNA in early diagnosis of chronic illness including cardiovascular and neurodegenerative diseases [58-60]. Therefore, pharmaceutical companies are very interested in developing a diagnostic kit for several types of cancer and chronic diseases [61].

According to the last version (21st) of miRNA database (miRBase) [62], there are 1881 human sequences identified and the list is still growing; Friedländer et al., employing an innovative computational method, reported 2469 novel human miRNA candidates [63]. Therefore, researchers have used two experimental strategies to find the "needle in a haystack": using omics approaches (microarray or NGS) and/or preselecting miRNAs based on previous finding reported in animal models (Table 2 ). In this context, NGS offers several advantages; for instance, it does not require the knowledge of either miRNA target or specific probes or primers to discover new miRNAs [64]. Regarding diabetes, since 2010 several differentially regulated miRNAs have been identified in human biofluids from patients of impaired glucose tolerance (IGT)/impaired fasting glucose (IFG), DMT2 in comparison to healthy controls (Table 2).

Reviewing the articles published in the last five years, we have concluded that there is not an extensive overlap in the results of miRNAs identification associated with different conditions of diabetes patients. Only miR-126 and miR-144 have been proposed as biomarkers for diagnosing diabetes in more than one study [65-68]. Although these clinical studies used the same range of values for glucose tolerance test (the most important parameter for patients classification), there are other factors that could explain the differences: (1) clinical characteristics including obesity, age, year after diagnosis, and lipid profile [69]; (2) treatment with hypoglycaemic drugs such as metformin [66]; (3) ethnical origin such as Iraqis versus Swedes [70]; (4) technical aspects: types of biofluids, differences in miRNA microarrays companies (quantity and type of miRNA tested), and miRNA normalization methods.

Regarding diabetic cardiomyopathy, diagnosed patients with diastolic dysfunction share many clinical characteristics with diabetic patients reported in the clinical study for the identification of miRNAs related to diabetes (Table 2). However, at present there are no clinical trials reporting circulating miRNAs as a candidate for diabetic cardiomyopathy diagnosis. On the other hand, there is no concordance between the miRNA identified with diabetic cardiomyopathy in animal models and those identified in human biofluids, except miR-34a and miR-30d, which were identified first in human plasma and then their mechanism of action was studied at a preclinical level $[32,35]$. The diabetic complications have a slow but progressive negative manifestation in the target organs (kidney, liver, heart, and retina) with respect to the apparent stability of metabolic parameters (fasting glucose and glucose tolerance test). As diabetes is a multifactorial disease, care should be taken when enrolling the patients, by following strict definitions of the clinical characteristic of the diabetic complications. In a crosssectional study where DMT1 patients were classified in three groups according to their level of renal dysfunction by eGFR, good renal function $\geq 30 \mathrm{~mL} / \mathrm{min}$ of creatinine clearance, renal failure $<30 \mathrm{~mL} / \mathrm{min}$ of creatinine clearance, and healthy control, four miRNAs (miR-181, miR-326, miR-126, and miR573-3p) were identified in plasma that could be useful to predict the development of diabetic nephropathy [71].

The use of disease animal models is a powerful tool to select circulating miRNAs candidate for biomarker as it allows establishing a correlation between miRNAs associated with specific injured organ and the biofluid from early to advanced stages of disease development. For instance, Bellinger et al. found a concordance between the expression of miR-714, miR-1188, miR-1897-3p, miR-877, and miR-1224 and progression of acute kidney injury reflected in the plasma of a mouse model, proposing them as a promising predictor of kidney injury [72]. Acharya et al. reported serum miRNA signatures that predict the impact of radiation in animals that were exposed to sublethal and lethal doses of radiation, 24 hours after exposure [73], and Rotkrua et al. selected circulating miRNAs (miR-103, miR-107, miR-194, and miR210) as biomarkers for early detection of diffuse-type gastric cancer using a mouse model and compared expression of these miRNAs in tumour tissue and serum samples [74]. The strategy of using diabetic cardiomyopathy animal models to find a corelation of miRNAs expression between myocardium and biofluids would not only provide relevant information but also accelerate miRNA identification $[8,75]$.

Regarding methodological aspects, it has been established that using different evaluation techniques may yield variations in the end results. For this reason, in the last years many methodological studies have been published comparing different laboratory procedures: (1) sample collection, (2) total miRNA isolation, (3) miRNA profiling methods, including qRT-PCR, miRNA microarrays (GeneChip and miRCURY LNA), and NGS, and (4) criteria of data analysis including miRNA normalization method (spike-in or internal miRNA). Further, alternatives in miRNA procedures were elaborately discussed in an excellent review of Moldovan et al. [76].

In a future perspective, a consistent miRNA profiling is not enough to diagnose a disease; informatics algorithms such as naïve Bayes classifier, J48 Decision Trees, and support vector machines are also necessary to identify the best miRNA profiling (considering all clinical characteristics) to discriminate between diabetic patients, which of them 


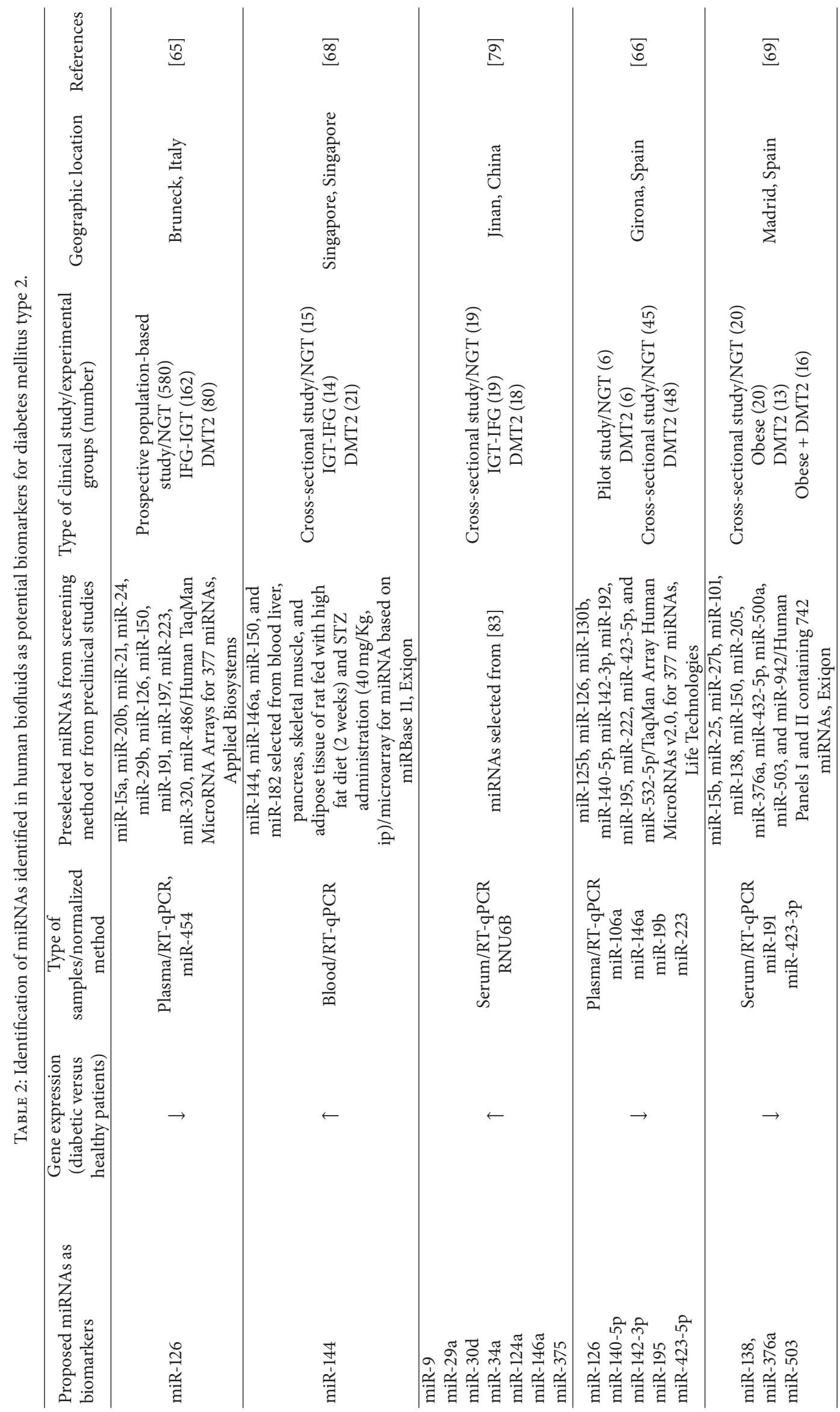




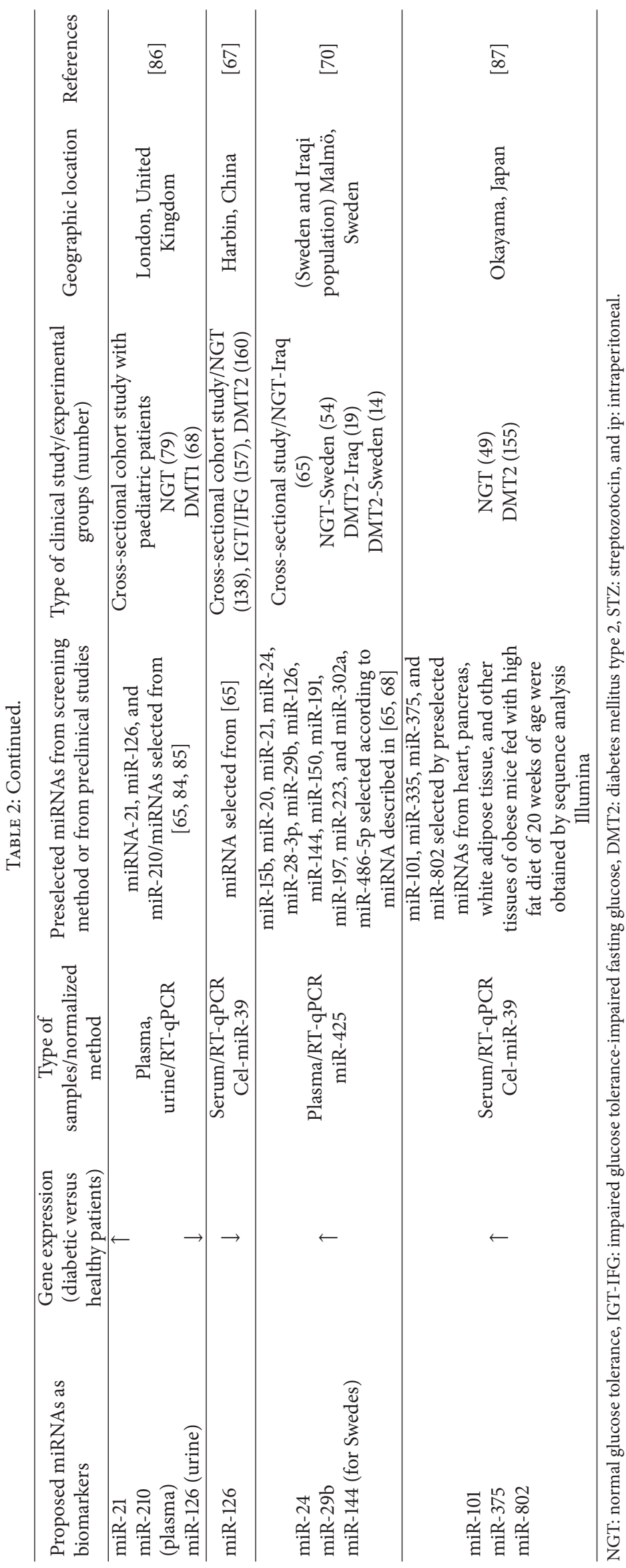




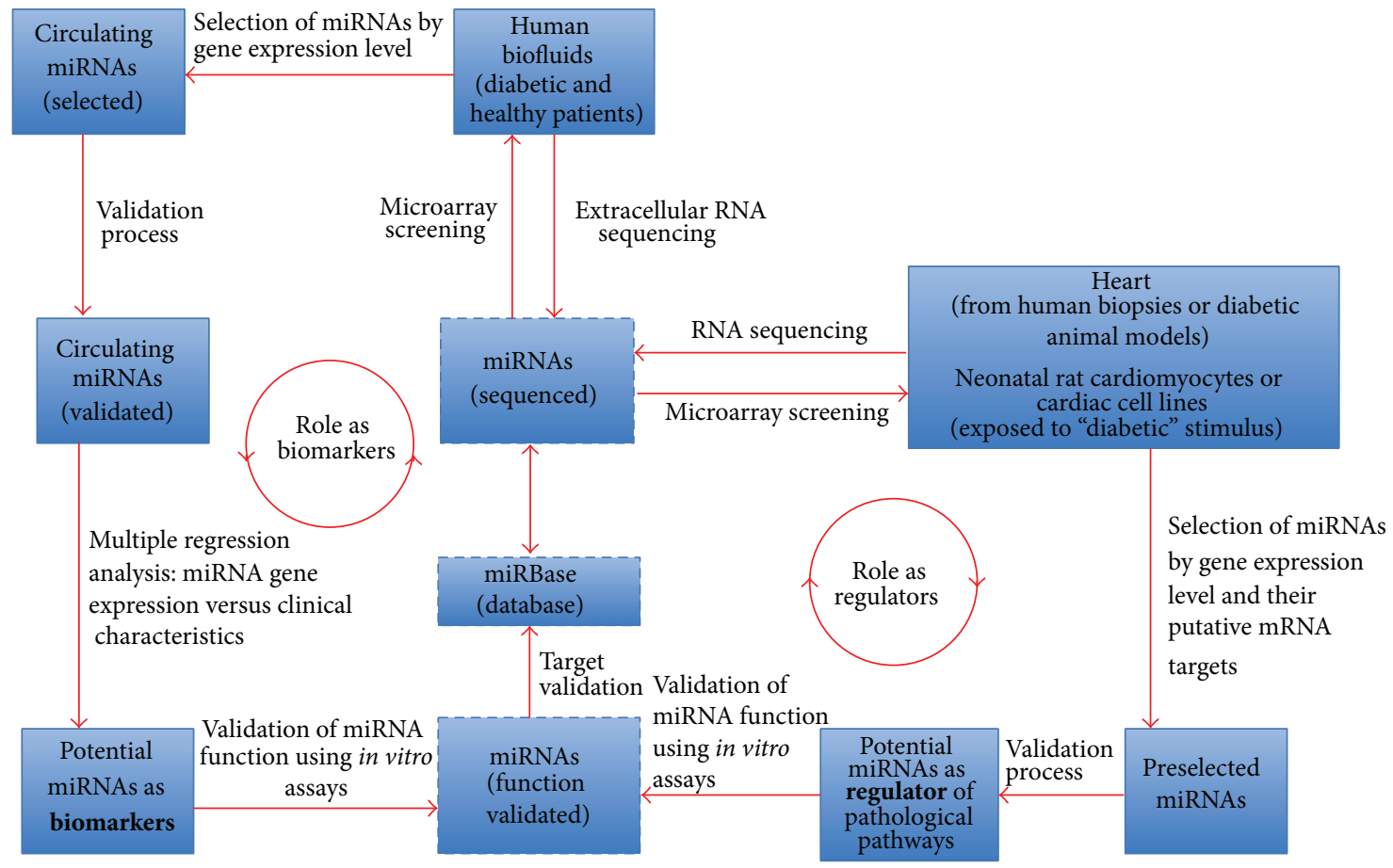

FIGURE 1: Experimental strategies for circulating microRNA identification as potential biomarkers.

have a high risk of developing diabetic cardiomyopathy. A summary of the experimental strategies for the identification of circulating miRNAs as biomarkers is described in Figure 1.

\section{Conclusions}

Diabetic cardiomyopathy progresses slowly and silently and is diagnosed (with the current detection procedures) only when the heart manifests a certain grade of dysfunction. This subclinical state of the illness becomes even more critical after an ischemic episode as it reduces the possibility to rescue the heart function to normal levels. At present, scientific evidence of the potential use of circulating miRNAs as biomarkers for cardiovascular diseases is increasing every day and is extensively backed up by the studies carried out by research groups of public and private institutions. The identification of miRNAs in diabetic patients "without" any secondary complications is relevant as it would allow narrowing down the miRNAs associated with specific diabetic complication. Regarding diabetic cardiomyopathy, the early detection using miRNAs biomarker could serve to intensify the antidiabetes treatment and cardioprophylactic therapies in patients with high risk of diabetes-derived diastolic dysfunction before it appears.

\section{Conflict of Interests}

The authors declare that there is no conflict of interests regarding the publication of this paper.

\section{Acknowledgments}

The authors thank Hollie Lawton for her contributions in the paper. This work was supported by Proyecto Mineduc-UDD
PMI 1204 "De la ciencia a la innovación en salud: adopción en la actividad clínica, nacional e internacional, de nuevos productos, procesos y prácticas de clase mundial, basados en investigación científica de la UDD y de terceros."

\section{References}

[1] IDF, IDF Diabetes Atlas, International Diabetes Federation, Brussels, Belgium, 6th edition, 2014.

[2] M. C. Blendea, S. I. McFarlane, E. R. Isenovic, G. Gick, and J. R. Sowers, "Heart disease in diabetic patients," Current Diabetes Reports, vol. 3, no. 3, pp. 223-229, 2003.

[3] A. I. Vinik and D. Ziegler, "Diabetic cardiovascular autonomic neuropathy," Circulation, vol. 115, no. 3, pp. 387-397, 2007.

[4] H. Bugger and C. Bode, "The vulnerable myocardium. Diabetic cardiomyopathy," Hämostaseologie, vol. 35, no. 1, pp. 17-24, 2015.

[5] A. M. From, C. L. Leibson, F. Bursi et al., "Diabetes in heart failure: prevalence and impact on outcome in the population," The American Journal of Medicine, vol. 119, no. 7, pp. 591-599, 2006.

[6] A. M. Shah, H. Uno, L. Køber et al., "The inter-relationship of diabetes and left ventricular systolic function on outcome after high-risk myocardial infarction," European Journal of Heart Failure, vol. 12, no. 11, pp. 1229-1237, 2010.

[7] S. Rubler, J. Dlugash, Y. Z. Yuceoglu, T. Kumral, A. W. Branwood, and A. Grishman, "New type of cardiomyopathy associated with diabetic glomerulosclerosis," The American Journal of Cardiology, vol. 30, no. 6, pp. 595-602, 1972.

[8] H. Bugger and E. D. Abel, "Rodent models of diabetic cardiomyopathy," Disease Models \& Mechanisms, vol. 2, no. 9-10, pp. 454466, 2009.

[9] H. Bugger and E. D. Abel, "Molecular mechanisms of diabetic cardiomyopathy," Diabetologia, vol. 57, no. 4, pp. 660-671, 2014. 
[10] T. Miki, S. Yuda, H. Kouzu, and T. Miura, "Diabetic cardiomyopathy: pathophysiology and clinical features," Heart Failure Reviews, vol. 18, no. 2, pp. 149-166, 2013.

[11] A. Dei Cas, V. Spigoni, V. Ridolfi, and M. Metra, "Diabetes and chronic heart failure: from diabetic cardiomyopathy to therapeutic approach," Endocrine, Metabolic \& Immune DisordersDrug Targets, vol. 13, no. 1, pp. 38-50, 2013.

[12] S. Boudina and E. D. Abel, "Diabetic cardiomyopathy, causes and effects," Reviews in Endocrine \& Metabolic Disorders, vol. 11, no. 1, pp. 31-39, 2010.

[13] V. Chavali, S. C. Tyagi, and P. K. Mishra, "Predictors and prevention of diabetic cardiomyopathy," Diabetes, Metabolic Syndrome and Obesity: Targets and Therapy, vol. 6, pp. 151-160, 2013.

[14] C. Teupe and C. Rosak, "Diabetic cardiomyopathy and diastolic heart failure-difficulties with relaxation," Diabetes Research and Clinical Practice, vol. 97, no. 2, pp. 185-194, 2012.

[15] J.-W. Ha, H.-C. Lee, E.-S. Kang et al., "Abnormal left ventricular longitudinal functional reserve in patients with diabetes mellitus: implication for detecting subclinical myocardial dysfunction using exercise tissue Doppler echocardiography," Heart, vol. 93, no. 12, pp. 1571-1576, 2007.

[16] J. M. McGavock, I. Lingvay, I. Zib et al., "Cardiac steatosis in diabetes mellitus: a $1 \mathrm{H}$-magnetic resonance spectroscopy study," Circulation, vol. 116, no. 10, pp. 1170-1175, 2007.

[17] P. Shah, B. G. Choi, and R. Mazhari, "Positron emission tomography for the evaluation and treatment of cardiomyopathy," Annals of the New York Academy of Sciences, vol. 1228, no. 1, pp. 137-149, 2011.

[18] S. Romano, M. Di Mauro, S. Fratini et al., "Early diagnosis of left ventricular diastolic dysfunction in diabetic patients: a possible role for natriuretic peptides," Cardiovascular Diabetology, vol. 9, article 89, 2010.

[19] K. Rahimi, D. Bennett, N. Conrad et al., "Risk prediction in patients with heart failure: a systematic review and analysis," JACC: Heart Failure, vol. 2, no. 5, pp. 440-446, 2014.

[20] A. Palazzuoli, S. Masson, C. Ronco, and A. Maisel, "Clinical relevance of biomarkers in heart failure and cardiorenal syndrome: the role of natriuretic peptides and troponin," Heart Failure Reviews, vol. 19, no. 2, pp. 267-284, 2014.

[21] E. C. Lai, "Two decades of miRNA biology: lessons and challenges," $R N A$, vol. 21, no. 4, pp. 675-677, 2015.

[22] M. Asrih and S. Steffens, "Emerging role of epigenetics and miRNA in diabetic cardiomyopathy," Cardiovascular Pathology, vol. 22, no. 2, pp. 117-125, 2013.

[23] V. K. Cortessis, D. C. Thomas, A. Joan Levine et al., "Environmental epigenetics: prospects for studying epigenetic mediation of exposure-response relationships," Human Genetics, vol. 131, no. 10, pp. 1565-1589, 2012.

[24] V. Ambros, "The functions of animal microRNAs," Nature, vol. 431, no. 7006, pp. 350-355, 2004.

[25] W. Filipowicz, S. N. Bhattacharyya, and N. Sonenberg, "Mechanisms of post-transcriptional regulation by microRNAs: are the answers in sight?" Nature Reviews Genetics, vol. 9, no. 2, pp. 102114, 2008.

[26] H. Lu, R. J. Buchan, and S. A. Cook, "MicroRNA-223 regulates Glut4 expression and cardiomyocyte glucose metabolism," Cardiovascular Research, vol. 86, no. 3, pp. 410-420, 2010.

[27] H. El Azzouzi, S. Leptidis, E. Dirkx et al., "The hypoxia-inducible microRNA cluster miR-199a approximately 214 targets myocardial PPARdelta and impairs mitochondrial fatty acid oxidation," Cell Metabolism, vol. 18, no. 3, pp. 341-354, 2013.
[28] Y. Duan, B. Zhou, H. Su, Y. Liu, and C. Du, "MiR-150 regulates high glucose-induced cardiomyocyte hypertrophy by targeting the transcriptional co-activator p300," Experimental Cell Research, vol. 319, no. 3, pp. 173-184, 2013.

[29] B. Feng, S. Chen, B. George, Q. Feng, and S. Chakrabarti, "miR133a regulates cardiomyocyte hypertrophy in diabetes," Diabetes/Metabolism Research and Reviews, vol. 26, no. 1, pp. 40-49, 2010.

[30] E. Shen, X. Diao, X. Wang, R. Chen, and B. Hu, "MicroRNAs involved in the mitogen-activated protein kinase cascades pathway during glucose-induced cardiomyocyte hypertrophy," The American Journal of Pathology, vol. 179, no. 2, pp. 639-650, 2011.

[31] X. Diao, E. Shen, X. Wang, and B. Hu, "Differentially expressed microRNAs and their target genes in the hearts of streptozotocin-induced diabetic mice," Molecular Medicine Reports, vol. 4, no. 4, pp. 633-640, 2011.

[32] F. Zhao, B. Li, Y.-Z. Wei et al., "MicroRNA-34a regulates high glucose-induced apoptosis in H9c2 cardiomyocytes," Journal of Huazhong University of Science and Technology-Medical Science, vol. 33, no. 6, pp. 834-839, 2013.

[33] X.-Y. Yu, Y.-H. Song, Y.-J. Geng et al., "Glucose induces apoptosis of cardiomyocytes via microRNA-1 and IGF-1," Biochemical and Biophysical Research Communications, vol. 376, no. 3, pp. 548-552, 2008.

[34] S. S. Yildirim, D. Akman, D. Catalucci, and B. Turan, "Relationship between downregulation of miRNAs and increase of oxidative stress in the development of diabetic cardiac dysfunction: junctin as a target protein of miR-1," Cell Biochemistry and Biophysics, vol. 67, no. 3, pp. 1397-1408, 2013.

[35] X. Li, N. Du, Q. Zhang et al., "MicroRNA-30d regulates cardiomyocyte pyroptosis by directly targeting foxo3a in diabetic cardiomyopathy," Cell Death and Disease, vol. 5, article e1479, 2014.

[36] X. H. Wang, R. Z. Qian, W. Zhang, S. F. Chen, H. M. Jin, and R. M. Hu, "MicroRNA-320 expression in myocardial microvascular endothelial cells and its relationship with insulin-like growth factor-1 in type 2 diabetic rats," Clinical and Experimental Pharmacology and Physiology, vol. 36, no. 2, pp. 181-188, 2009.

[37] S. K. Panguluri, J. Tur, K. C. Chapalamadugu, C. Katnik, J. Cuevas, and S. M. Tipparaju, "MicroRNA-301a mediated regulation of Kv4.2 in diabetes: identification of key modulators," PLoS ONE, vol. 8, no. 4, article e60545, 2013.

[38] W. A. Baseler, D. Thapa, R. Jagannathan, E. R. Dabkowski, T. L. Croston, and J. M. Hollander, "miR-141 as a regulator of the mitochondrial phosphate carrier (Slc25a3) in the type 1 diabetic heart," The American Journal of Physiology-Cell Physiology, vol. 303, no. 12, pp. C1244-C1251, 2012.

[39] Y. Kuwabara, T. Horie, O. Baba et al., "MicroRNA-451 exacerbates lipotoxicity in cardiac myocytes and high-fat dietinduced cardiac hypertrophy in mice through suppression of the LKB1/AMPK pathway," Circulation Research, vol. 116, no. 2, pp. 279-288, 2015.

[40] S. Ong and J. C. Wu, "Exosomes as potential alternatives to stem cell therapy in mediating cardiac regeneration," Circulation Research, vol. 117, no. 1, pp. 7-9, 2015.

[41] Z. A. Malik, K. S. Kott, A. J. Poe et al., "Cardiac myocyte exosomes: stability, HSP60, and proteomics," The American Journal of Physiology-Heart and Circulatory Physiology, vol. 304, no. 7, pp. H954-H965, 2013. 
[42] N. Gennebäck, U. Hellman, L. Malm et al., "Growth factor stimulation of cardiomyocytes induces changes in the transcriptional contents of secreted exosomes," Journal of Extracellular Vesicles, vol. 2, 2013.

[43] A. Waldenström, N. Gennebäck, U. Hellman, and G. Ronquist, "Cardiomyocyte microvesicles contain DNA/RNA and convey biological messages to target cells," PLoS ONE, vol. 7, no. 4, Article ID e34653, 2012.

[44] C. Bang, S. Batkai, S. Dangwal et al., "Cardiac fibroblast-derived microRNA passenger strand-enriched exosomes mediate cardiomyocyte hypertrophy," The Journal of Clinical Investigation, vol. 124, no. 5, pp. 2136-2146, 2014.

[45] S. Rani, "MicroRNA profiling of exosomes isolated from biofluids and conditioned media," Methods in Molecular Biology, vol. 1182, pp. 131-144, 2014.

[46] K. C. Vickers, B. T. Palmisano, B. M. Shoucri, R. D. Shamburek, and A. T. Remaley, "MicroRNAs are transported in plasma and delivered to recipient cells by high-density lipoproteins," Nature Cell Biology, vol. 13, no. 4, pp. 423-435, 2011.

[47] F. Spillmann, S. van Linthout, and C. Tschöpe, "Cardiac effects of HDL and its components on diabetic cardiomyopathy," Endocrine, Metabolic \& Immune Disorders-Drug Targets, vol. 12, no. 2, pp. 132-147, 2012.

[48] J. F. Aranda, J. Madrigal-Matute, N. Rotllan, and C. FernándezHernando, "MicroRNA modulation of lipid metabolism and oxidative stress in cardiometabolic diseases," Free Radical Biology and Medicine, vol. 64, pp. 31-39, 2013.

[49] G. Meister, "Argonaute proteins: functional insights and emerging roles," Nature Reviews Genetics, vol. 14, no. 7, pp. 447-459, 2013.

[50] J. D. Arroyo, J. R. Chevillet, E. M. Kroh et al., "Argonaute2 complexes carry a population of circulating microRNAs independent of vesicles in human plasma," Proceedings of the National Academy of Sciences of the United States of America, vol. 108, no. 12, pp. 5003-5008, 2011.

[51] R. C. Friedman, K. K.-H. Farh, C. B. Burge, and D. P. Bartel, "Most mammalian mRNAs are conserved targets of microRNAs," Genome Research, vol. 19, no. 1, pp. 92-105, 2009.

[52] J. P. Mehta, "Sequencing small RNA: introduction and data analysis fundamentals," Methods in Molecular Biology, vol. 1182, pp. 93-103, 2014.

[53] P. Alexiou, M. Maragkakis, G. L. Papadopoulos, V. A. Simmosis, L. Zhang, and A. G. Hatzigeorgiou, "The DIANA-mirExTra web server: from gene expression data to microRNA function," PLoS ONE, vol. 5, no. 2, Article ID e9171, 2010.

[54] M. Kunz, K. Xiao, C. Liang et al., "Bioinformatics of cardiovascular miRNA biology," Journal of Molecular and Cellular Cardiology, 2014.

[55] H. Dweep, C. Sticht, P. Pandey, and N. Gretz, "MiRWalkdatabase: prediction of possible miRNA binding sites by 'walking' the genes of three genomes," Journal of Biomedical Informatics, vol. 44, no. 5, pp. 839-847, 2011.

[56] S. S. C. Chim, T. K. F. Shing, E. C. W. Hung et al., "Detection and characterization of placental microRNAs in maternal plasma," Clinical Chemistry, vol. 54, no. 3, pp. 482-490, 2008.

[57] C. H. Lawrie, S. Gal, H. M. Dunlop et al., "Detection of elevated levels of tumour-associated microRNAs in serum of patients with diffuse large B-cell lymphoma," British Journal of Haematology, vol. 141, no. 5, pp. 672-675, 2008.

[58] M. A. Cortez and G. A. Calin, "MicroRNA identification in plasma and serum: a new tool to diagnose and monitor diseases," Expert Opinion on Biological Therapy, vol. 9, no. 6, pp. 703-711, 2009.

[59] S. P. Romaine, M. Tomaszewski, G. Condorelli, and N. J. Samani, "MicroRNAs in cardiovascular disease: an introduction for clinicians," Heart, vol. 101, no. 12, pp. 921-928, 2015.

[60] L. Tan, J.-T. Yu, and L. Tan, "Causes and consequences of microRNA dysregulation in neurodegenerative diseases," Molecular Neurobiology, vol. 51, no. 3, pp. 1249-1262, 2015.

[61] G. S. Mack, "MicroRNA gets down to business," Nature Biotechnology, vol. 25, no. 6, pp. 631-638, 2007.

[62] miRBase, 21st version, http://www.mirbase.org/.

[63] M. R. Friedländer, E. Lizano, A. J. S. Houben et al., "Evidence for the biogenesis of more than 1,000 novel human microRNAs," Genome Biology, vol. 15, no. 4, article R57, 2014.

[64] C. C. Pritchard, H. H. Cheng, and M. Tewari, "MicroRNA profiling: approaches and considerations," Nature Reviews Genetics, vol. 13, no. 5, pp. 358-369, 2012.

[65] A. Zampetaki, S. Kiechl, I. Drozdov et al., "Plasma MicroRNA profiling reveals loss of endothelial miR-126 and other microRNAs in type 2 diabetes," Circulation Research, vol. 107, no. 6, pp. 810-817, 2010.

[66] F. J. Ortega, J. M. Mercader, J. M. Moreno-Navarrete et al., "Profiling of circulating microRNAs reveals common microRNAs linked to type 2 diabetes that change with insulin sensitization," Diabetes Care, vol. 37, no. 5, pp. 1375-1383, 2014.

[67] Y. Liu, G. Gao, C. Yang et al., "The role of circulating microRNA126 (miR-126): a novel biomarker for screening prediabetes and newly diagnosed type 2 diabetes mellitus," International Journal of Molecular Sciences, vol. 15, no. 6, pp. 10567-10577, 2014.

[68] D. S. Karolina, A. Armugam, S. Tavintharan et al., "MicroRNA 144 impairs insulin signaling by inhibiting the expression of insulin receptor substrate 1 in type 2 diabetes mellitus," PLoS ONE, vol. 6, no. 8, Article ID e22839, 2011.

[69] N. Pescador, M. Pérez-Barba, J. M. Ibarra, A. Corbatón, M. T. Martínez-Larrad, and M. Serrano-Ríos, "Serum circulating microRNA profiling for identification of potential type 2 diabetes and obesity biomarkers," PLoS ONE, vol. 8, no. 10, Article ID e77251, 2013.

[70] X. Wang, J. Sundquist, B. Zöller et al., "Determination of 14 circulating microRNAs in Swedes and Iraqis with and without diabetes mellitus type 2," PLoS ONE, vol. 9, no. 1, Article ID e86792, 2014.

[71] R. Bijkerk, J. M. Duijs, M. Khairoun et al., "Circulating MicroRNAs associate with diabetic nephropathy and systemic microvascular damage and normalize after simultaneous pancreas-kidney transplantation," American Journal of Transplantation, vol. 15, no. 4, pp. 1081-1090, 2015.

[72] M. A. Bellinger, J. S. Bean, M. A. Rader et al., "Concordant changes of plasma and kidney microRNA in the early stages of acute kidney injury: time course in a mouse model of bilateral renal ischemia-reperfusion," PLoS ONE, vol. 9, no. 4, Article ID e93297, 2014.

[73] S. S. Acharya, W. Fendler, J. Watson et al., "Serum microRNAs are early indicators of survival after radiation-induced hematopoietic injury," Science Translational Medicine, vol. 7, no. 287, Article ID 287ra269, 2015.

[74] P. Rotkrua, S. Shimada, K. Mogushi, Y. Akiyama, H. Tanaka, and Y. Yuasa, "Circulating microRNAs as biomarkers for early detection of diffuse-type gastric cancer using a mouse model," British Journal of Cancer, vol. 108, no. 4, pp. 932-940, 2013. 
[75] S. D. Calligaris, M. Lecanda, F. Solis et al., "Mice longterm high-fat diet feeding recapitulates human cardiovascular alterations: an animal model to study the early phases of diabetic cardiomyopathy," PLoS ONE, vol. 8, no. 4, Article ID e60931, 2013.

[76] L. Moldovan, K. E. Batte, J. Trgovcich, J. Wisler, C. B. Marsh, and M. Piper, "Methodological challenges in utilizing miRNAs as circulating biomarkers," Journal of Cellular and Molecular Medicine, vol. 18, no. 3, pp. 371-390, 2014.

[77] B. Yang, H. Lin, J. Xiao et al., "The muscle-specific microRNA miR-1 regulates cardiac arrhythmogenic potential by targeting GJA1 and KCNJ2," Nature Medicine, vol. 13, no. 4, pp. 486-491, 2007.

[78] J. Xiao, X. Luo, H. Lin et al., "MicroRNA miR-133 represses HERG $\mathrm{K}^{+}$channel expression contributing to QT prolongation in diabetic hearts," The Journal of Biological Chemistry, vol. 282, pp. 12363-12367, 2007.

[79] L. Kong, J. Zhu, W. Han et al., "Significance of serum microRNAs in pre-diabetes and newly diagnosed type 2 diabetes: a clinical study," Acta Diabetologica, vol. 48, no. 1, pp. 61-69, 2011.

[80] E. van Rooij, L. B. Sutherland, N. Liu et al., "A signature pattern of stress-responsive microRNAs that can evoke cardiac hypertrophy and heart failure," Proceedings of the National Academy of Sciences of the United States of America, vol. 103, no. 48, pp. 18255-18260, 2006.

[81] Z. Lu, Y. Li, A. Takwi et al., "miR-301a as an NF-kappaB activator in pancreatic cancer cells," The EMBO Journal, vol. 30, no. 1, pp. 57-67, 2011.

[82] B. K. Panama, D. Latour-Villamil, G. P. Farman et al., "Nuclear factor $\kappa \mathrm{b}$ downregulates the transient outward potassium current Ito,f through control of KChIP2 expression," Circulation Research, vol. 108, no. 5, pp. 537-543, 2011.

[83] A. K. Pandey, P. Agarwal, K. Kaur, and M. Datta, "MicroRNAs in diabetes: tiny players in big disease," Cellular Physiology and Biochemistry, vol. 23, no. 4-6, pp. 221-232, 2009.

[84] S. Greco, P. Fasanaro, S. Castelvecchio et al., "MicroRNA dysregulation in diabetic ischemic heart failure patients," Diabetes, vol. 61, no. 6, pp. 1633-1641, 2012.

[85] X. Zhong, A. C. K. Chung, H. Y. Chen et al., "MiR-21 is a key therapeutic target for renal injury in a mouse model of type 2 diabetes," Diabetologia, vol. 56, no. 3, pp. 663-674, 2013.

[86] J. Osipova, D.-C. Fischer, S. Dangwal et al., "Diabetes-associated MicroRNAs in pediatric patients with type 1 diabetes mellitus: a cross-sectional cohort study," Journal of Clinical Endocrinology and Metabolism, vol. 99, no. 9, pp. E1661-E1665, 2014.

[87] C. Higuchi, A. Nakatsuka, J. Eguchi et al., "Identification of circulating miR-101, miR-375 and miR-802 as biomarkers for type 2 diabetes," Metabolism, vol. 64, no. 4, pp. 489-497, 2015. 


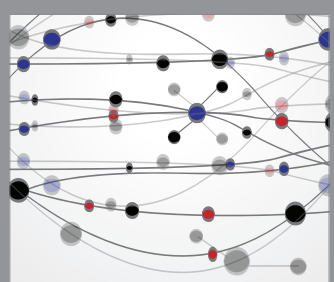

The Scientific World Journal
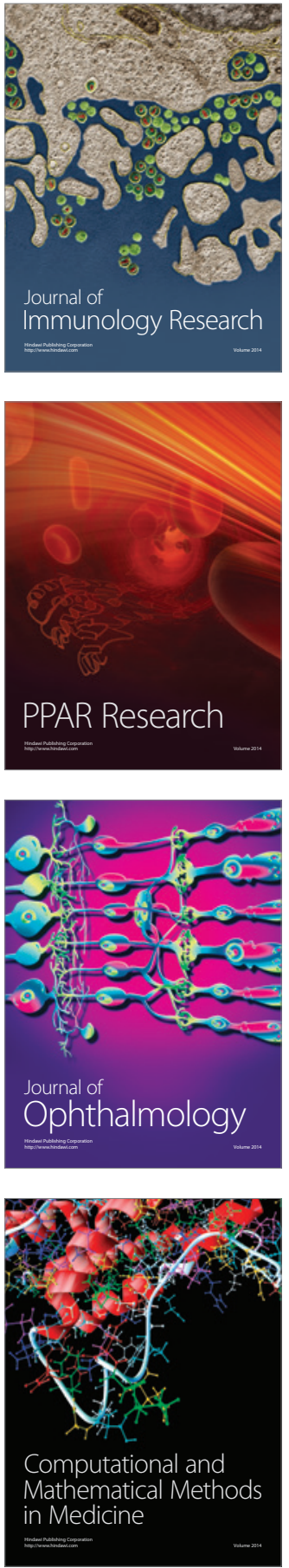

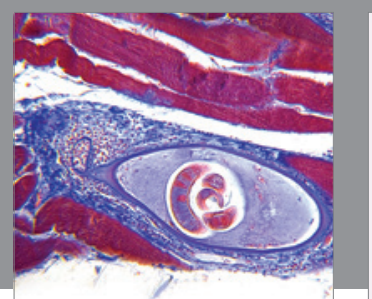

Gastroenterology Research and Practice

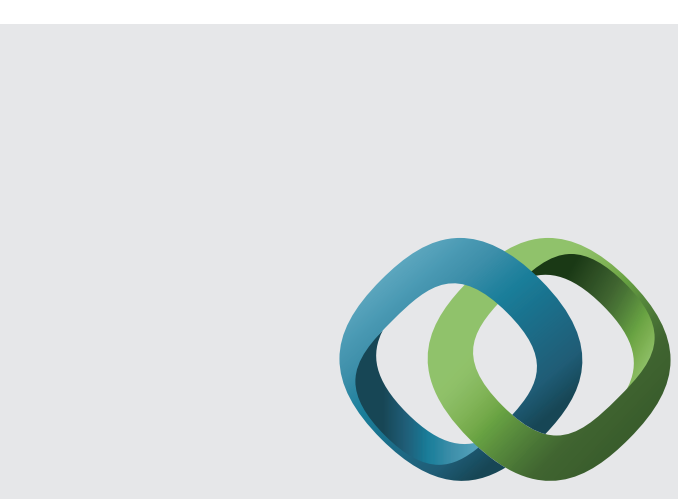

\section{Hindawi}

Submit your manuscripts at

http://www.hindawi.com
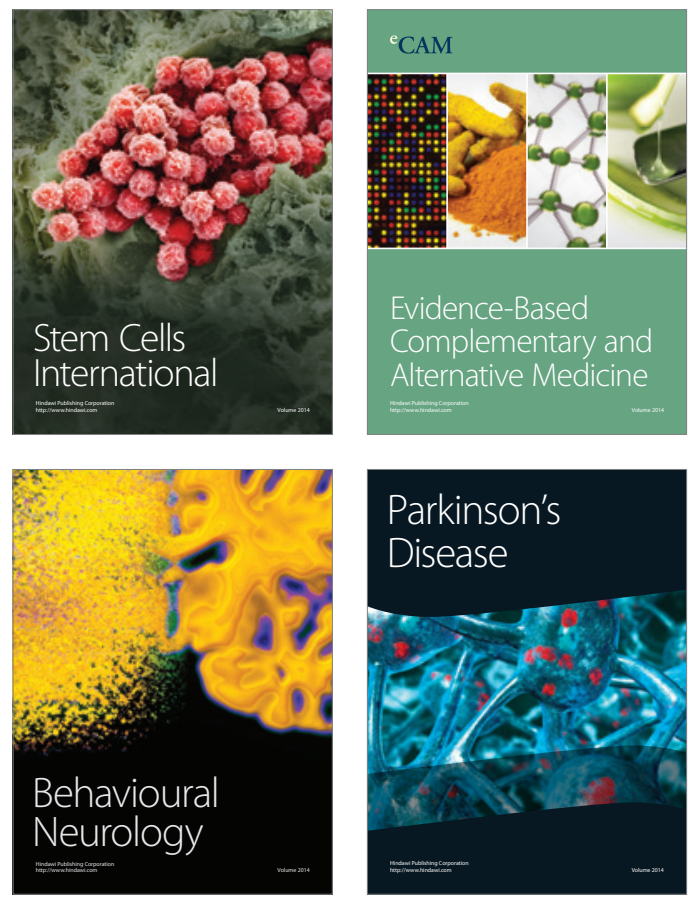
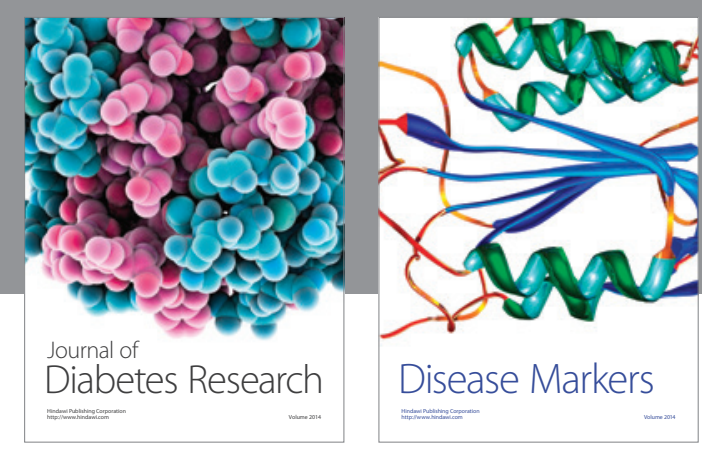

Disease Markers
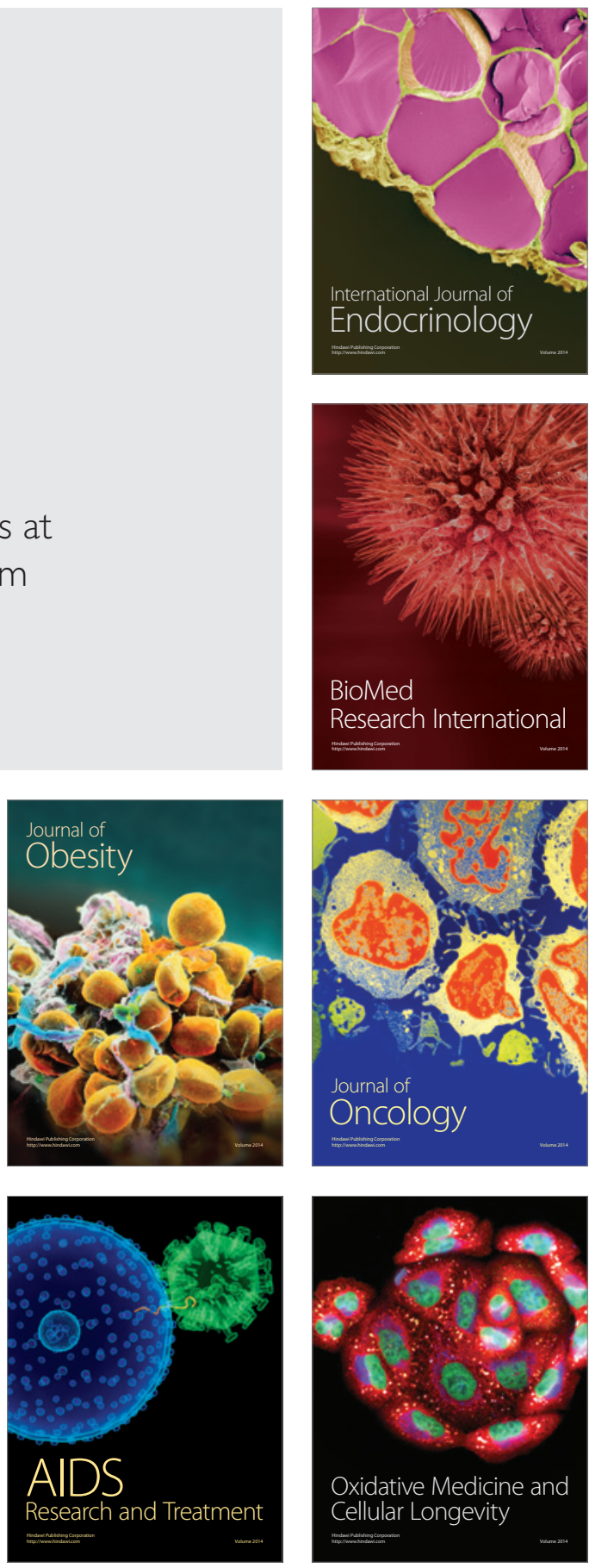